\title{
1 ESCRT-III accumulates in micronuclei with ruptured nuclear envelopes
}

2 Running title: ESCRT-III accumulations in micronuclei

3 Jessica Willan ${ }^{1,4 \pi}$, Alexa Cleasby ${ }^{1 \pi}$, Neftali Flores-Rodriguez²n, Flavia Stefani ${ }^{3}$, Cinzia

4 Rinaldo ${ }^{5}$, Alessandra Pisciottani ${ }^{5}$, Emma Grant ${ }^{1,4}$, Philip Woodman ${ }^{3 \S}$, Helen Bryant ${ }^{4 \S}$

5 and Barbara Ciani ${ }^{1, \S}$

61 Department of Chemistry, Centre for Membrane Interactions and Dynamics

7 (CMIAD), Krebs Institute, University of Sheffield, Brook Hill, Sheffield S3 7HF, United

8 Kingdom; 2 The University of Sydney, Australian Centre for Microscopy and

9 Microanalysis (Sydney, Australia); 3 University of Manchester, School of Biological

10 Sciences, Faculty and Biology Medicine and Health, Manchester Academic Health

11 Science Centre (Manchester, United Kingdom); 4 Academic Unit of Molecular

12 Oncology, Sheffield Institute for Nucleic Acids (SInFoNiA), Department of Oncology

13 and Metabolism, University of Sheffield, Beech Hill Road, Sheffield S10 2RX, United

14 Kingdom; 5 IBMP-CNR, Universita' degli Studi di Roma "La Sapienza", Rome, Italy.

16 "These authors equally contributed to this work

$17 \S$ Corresponding authors 


\section{Abstract}

Micronuclei represent the cellular attempt to compartmentalize DNA to maintain

21 genomic integrity threatened by mitotic errors and genotoxic events. Micronuclei show aberrant nuclear envelopes that collapse, generating damaged DNA and promoting complex genome alterations. However, ruptured micronuclei also provide a pool of cytosolic DNA that stimulates anti-tumour immunity, revealing the complexity of micronuclei impact on tumour progression.

The ESCRT-III complex ensures nuclear envelope (NE) resealing during late mitosis and NE repair in interphase. Therefore, ESCRT-III activity maybe crucial for maintaining the integrity of other genomic structures enclosed by a nuclear envelope. ESCRT-III activity at the nuclear envelope is coordinated by the subunit CHMP7.

31 We show that CHMP7 and ESCRT-III protects against the genomic instability associated with micronuclei formation. Loss of ESCRT-III activity increases the population of micronuclei with ruptured nuclear envelopes, in interphase cells.

34 Surprisingly, ESCRT-III is retained at acentric micronuclei suggesting that ESCRT-III cannot repair these structures. Depletion of CHMP7 expression removes ESCRT-III

36 accumulations at ruptured micronuclei, and removes the population of micronuclei

37 with damaged DNA also containing a sensor for cytosolic DNA.

38 Thus, ESCRT-III activity appears to protect from the consequence of genomic

39 instability in a dichotomous fashion. Membrane repair activity prevents the 
40 occurrence of $\mathrm{MN}$ with weak envelopes; conversely, aberrant membrane

41 remodelling at micronuclei generates a steady state pool of cytosolic DNA that may contribute to sustaining pro-inflammatory pathways in cancer cells.

\section{Keywords}

Micronuclei, genomic instability, cytosolic DNA, nuclear envelope, ESCRT-III

\section{Introduction}

Micronuclei are cytosolic chromatin structures that are compartmentalized by a

49 nuclear envelope (NE). They are a measure of chromosome instability and thus are

50 hallmarks of cancer cells. Micronuclei originate during mitosis, either because whole

51 chromosomes separate aberrantly, or because DNA damage generates

52 chromosomal fragments that lack centromeres and thus fail to align at the

53 metaphase plate ${ }^{1}$. The resulting structures rebuild their own NE away from the main

54 chromatin mass. Micronuclei persist through multiple cell divisions, but their NE can collapse within the $\mathrm{G} 2$ phase of the cell cycle ${ }^{2}$. The cause of such collapse remains

56 unclear, but correlates with a lack of nuclear lamina integrity ${ }^{2}$. Such loss of

57 micronuclear compartmentalization causes cytosolic enzymes to enter the 58 micronucleus, generating further DNA damage and chromosome pulverization ${ }^{3}$. A 59 micronucleus can reincorporate in the primary nucleus creating the conditions for

60 DNA fragments to rejoin the main genome at random locations (chromothripsis). 
61 Therefore, an intact NE around a micronucleus maintains the integrity of its genetic material ${ }^{4-6}$ and thereby protects against chromothripsis.

63 NE integrity at the primary nucleus is ensured by ESCRT-III, a universal membrane-

64 remodeling complex. Specifically, ESCRT-III seals nuclear membranes during late mitosis and repairs mechanical rupture of the NE during interphase ${ }^{7-10}$. Core ESCRTIII subunits, including the critical membrane-deforming polymer CHMP4B (Charged Multivesicular body Protein 4B), are recruited by $\mathrm{CHMP7}^{11}$, a specialized ESCRT-III subunit that is targeted to NE gaps by associating with the chromatin binding

69 protein LEM2(ref.12). ESCRT-III seals these gaps by supporting reverse-topology membrane scission ${ }^{13}$. ESCRT-III activity at the NE is short-lived, and is regulated by the AAA ATPase, VPS4. VPS4 remodels ESCRT-III to drive membrane scission, and also recycles ESCRT-III subunits back into the cytosol ${ }^{13}$. Loss of micronuclear compartmentalization exposes of DNA to the cytosol, which drives protective immune responses ${ }^{14}$. Cytosolic DNA is recognized as foreign by innate immune pathways involving cyclic GAMP synthase (cGAS) ${ }^{15}$. cGAS binds to specific secondary structures within exposed double and single-stranded DNA and stimulates the production of $2^{\prime}-5^{\prime}$ cyclic GMP-AMP (cGAMP) ${ }^{16}$.

Here we present how ESCRT-III protects the genome from the instability generated by micronuclei. We show that ESCRT-III and VPS4 support micronuclear envelope membrane integrity, mirroring their role in maintaining the primary $\mathrm{NE}^{17}$. We also 
81 show that ESCRT-III activity is aberrant at acentric collapsed micronuclei in

82 unperturbed cancer cells. We interpret this aberrant activity of ESCRT-III as

83 necessary for maintaining a population of ruptured, cGAS-enriched micronuclei.

84 Consistent with such a role, this pool of collapsed micronuclei containing ESCRT-III, cGAS and ssDNA is maintained by impairing ESCRT-III recycling and is removed by preventing ESCRT-III recruitment at the nuclear envelope.

\section{Results}

Depletion of CHMP7 or VPS4 compromise the nuclear envelope but damage DNA in different fashion.

91 ESCRT-III helps to re-form the NE during cell division and repairs the NE during

92 interphase $7,9,10,18$. To further understand the impact of ESCRT-III on nuclear envelope

93 integrity in micronuclei, we first performed a detailed analysis of the phenotypes

94 observed at the interphase nuclei when ESCRT-III activity is impaired. We depleted

95 CHMP7 (Supplemental Figure 1A, B), which initiates NE-associated ESCRT-III 96 assembly ${ }^{11}$, and VPS4 (Supplemental Figure 1C and see Methods), which regulates 97 ESCRT-III-mediated membrane remodeling activity ${ }^{17}$.

98 Depletion of CHMP7 or VPS4 increased the frequency of multinucleated cells 99 (Supplemental Figure 1D) and increased the proportion of deformed interphase 
on the role of ESCRT-III in NE sealing, we observed defects in NE structure within

$109<1 \%$ of interphase untreated or control-treated cells. CHMP7 accumulations in

110 VPS4 depleted cells also contained CHMP4B (Figure 1F, H), whilst CHMP4B was

111 absent when cells were co-depleted for VPS4 and CHMP7 (Figure 1H), in agreement

112 with CHMP7 coordinating the recruitment of CHMP4B at the nuclear envelope.

113 Consistent with their function at the NE, CHMP7 or VPS4 depletion caused a loss of

114 nuclear compartmentalization, as revealed by the presence in the cytoplasm of

115 ProMyelocytic Leukemia (PML) bodies $^{20}$ (Supplemental Figure 2A, B). Additionally,

116 h-TERT human fibroblasts depleted of CHMP7 displayed an increased cytosolic

117 distribution of a general nuclear import marker (GFP-NLS, Supplemental Figure 2A,

118 C and Supplemental Figure 1F).

119 CHMP7 and VPS4 depletion generated substantial DNA damage as assessed by

120 staining with $\gamma \mathrm{H} 2 \mathrm{AX}$, a marker for double-strand breaks ${ }^{21,22}$. Upon CHMP7 depletion

121 (Supplemental Figure 2D), $\gamma \mathrm{H} 2 \mathrm{AX}$ stained abundant foci throughout the nucleus, 
122 which were large compared to the rare $\gamma \mathrm{H} 2 \mathrm{AX}$ foci seen in control cells

123 (Supplemental Figure 2E,F). Abundant $\gamma \mathrm{H} 2 \mathrm{AX}$ foci were also seen in VPS4 depleted

124 cells (Supplemental Figure 2E), nevertheless these were generally smaller than those seen in CHMP7 depleted cells (Supplemental Figure 2F) and, confined towards the nuclear periphery surrounding large CHMP7 accumulations (Supplemental Figure

$1272 \mathrm{G}$ top panels). CHMP7 foci in VPS4 depleted cells also labelled for the DNA repair

128 markers Rad51 and RPA (Supplemental Figure 2G, H), indicating the presence of single-stranded DNA (ssDNA) ${ }^{23}$.

130 Overall, these data reflect the difference between coordination of recruitment

131 (CHMP7) and dynamics of ESCRT-III assembly (VPS4) at the NE7,9,10. CHMP7

132 depleted cells are defective in nuclear envelope assembly and show widespread

133 membrane disorganization, rupture and DNA damage. In contrast, VPS4 depletion

134 induces aberrant ESCRT-III accumulations that associate with localized nuclear 135 envelope membrane enrichment and ssDNA.

137 Loss of ESCRT-III activity increases micronuclei with compromised envelope 138 membranes in interphase.

139 Cells displaying chromosomal instability are characterized by micronuclei, which are

140 generated when lagging chromosomes or chromosome fragments are enveloped

141 by NE membranes. Lagging DNA arises directly from kinetochore-microtubule 
142 attachment errors during mitosis (aneugenic mechanisms), as well as an indirect

143 consequence of unrepaired DNA damage during previous cell cycles that

144 generated acentric chromosome fragments (clastogenic mechanisms) $21,24,25$. Given

145 the roles of ESCRT-III at the nucleus, mitosis and cell division ${ }^{10,18,26,27}$, we assessed

146 the impact of impairing ESCRT-III function on the incidence of micronuclei with

147 ruptured envelopes.

148 Depletion of CHMP7 and/or VPS4 resulted in at least $\sim 2$-fold increase in the

149 population of cells containing micronuclei (Figure 2A,B) and a modest increase in

150 the number of micronuclei per micronucleated cell (Figure 2C). Micronuclei arising

151 from clastogenic events 28,29 are acentric, whilst micronuclei arising from aneugenic

152 events contain whole chromosomes and therefore contain centromeres.

153 Approximately $50 \%$ of micronuclei in control cells contained centromeres, as

154 assessed by staining for the centromeric marker CREST. This proportion changed

155 only slightly upon depletion of either CHMP7 or VPS4, albeit with opposite

156 tendencies (Figure 2D). Hence, the increase in micronuclei resulting from ESCRT-III

157 depletion most likely arises via a combination of aneugenic and clastogenic 158 mechanisms.

159 We then examined lamin B staining to assess the impact of ESCRT-III disruption on

160 the NE of micronuclei. Depletion of VPS4 or CHMP7 increased the proportion of

161 micronuclei in which the lamina was absent or discontinuous (Figure 2E;

162 Supplemental Fig 3A), recapitulating the phenotype observed at the primary 
nucleus. Micronuclei lacking nuclear pore complexes (NPCs) may be unable to

164 import lamins, and indeed the density of NPCs at micronuclei, assessed by mab414

166 (Figure 2F). Disruption of NE architecture can lead to invasion of the ER into the

167 micronucleus core ${ }^{2}$. Depletion of CHMP7 or VPS4 increased ER invasion, as

assessed by intense labeling of micronuclei with the lumenal ER protein PDI (Figure

$2 \mathrm{G}$ and $5 \mathrm{~A}, \mathrm{C})$. Moreover, fewer micronuclei in CHMP7 or VPS4 depleted cells

170 labeled for the soluble nuclear enzyme PARP1 (Figure 2H) or for GFP-NLS (Figure

$2 \mathrm{I}, \mathrm{J})$, indicating compromised compartmentalization. In summary, loss of ESCRT-III

172 function promotes formation micronuclei that largely show impaired NE integrity.

174 ESCRT-III accumulates within a subset of micronuclei.

175 Interestingly, we observed a significant enrichment of ESCRT-III proteins at the

176 nuclear envelope of micronuclei in unperturbed cells. Therefore, we examined the

177 morphology of the structures that displayed localization of endogenous ESCRT-III.

178 ESCRT-III residency at the reforming NE of the primary nucleus is dynamic and

179 short-lived ${ }^{10}$, such that large ESCRT-III accumulations are very rarely detected at the

180 interphase nucleus at steady state but are observed in $>80 \%$ cells when VPS4 is

181 depleted (Figure 1H). To our surprise, however, we found that at micronuclei the

182 situation is strikingly different. Specifically, intense CHMP7 labeling (CHMP7+ve) 
was found on $\sim 20-25 \%$ of micronuclei in non-treated or control-treated cells (Figure

184 3A-C), revealing an accumulation never seen at the primary nucleus and pointing towards a potential dysfunction of ESCRT-III at micronuclei. CHMP7 labeling of micronuclei was observed at a similar frequency across several cancer cell lines

187 (Figure 3B). CHMP7-labelled micronuclei also stained strongly for CHMP4B (Figure

3A, D). Whilst CHMP7 labeling was confined to peripheral puncta upon VPS4 KD

189 (Supplemental Figure 2G), STED microscopy revealed that in most (32/35)

190 micronuclei that labeled for ESCRT-III, the staining localized deep within the

191 micronucleus core (Figure 3D, E).

192 When VPS4 was depleted, the population of CHMP7-enriched micronuclei rose to

$193 \sim 60 \%$ (Figure 3D). Thus, an additional pool of ESCRT-III associates dynamically

194 ESCRT-III with micronuclei, consistent with ESCRT-III mediated repair (Figure 2).

195 Recruitment of CHMP4B to micronuclei, depended on CHMP7 (Figure 3C) both in

196 the presence and absence of VPS4. Hence, both dynamic and persistent pools of

197 ESCRT-III at micronuclei assemble in similar order to that observed at the primary 198 nucleus.

200 ESCRT-IIl enriched micronuclei lack NE integrity and nuclear compartmentalization.

201 Overall, these data suggest that ESCRT-III associates with micronuclei dynamically,

202 presumably as part of a repair process, but also point towards a dramatic retention 
bioRxiv preprint doi: https://doi.org/10.1101/476630; this version posted December 1, 2018. The copyright holder for this preprint (which

was not certified by peer review) is the author/funder, who has granted bioRxiv a license to display the preprint in perpetuity. It is made available under aCC-BY-NC-ND 4.0 International license.

203 of ESCRT-III under normal conditions at a subset of micronuclei. The deep

204 penetration into micronuclei of ESCRT-III accumulations potentially points to gross

205 structural defects in these structures. Indeed, whilst most CHMP7-negative

206 micronuclei had an intact (i.e. 'continuous') lamina, the lamina was either absent

207 from or discontinuous in virtually all CHMP7-positive micronuclei (Figure 4A,B and

208 Supplementary Figure 3A). As expected, when a discontinuous Lamin B was

209 present, CHMP7 occupied the gaps in Lamin B staining (Figure 4A).

211 We then quantified CHMP7-positive micronuclei according to the density of nuclear

212 pore complexes (NPCs). Most CHMP7-positive micronuclei did not contain, or had

213 reduced density, of NPCs compared to CHMP7-negative micronuclei (Figure 4C, D

214 and Supplemental Figure 3B). CHMP7-positive micronuclei also rarely contained

215 PARP1, in contrast to CHMP7-negative micronuclei (Figure 4E, F), highlighting a

216 defect in compartmentalization. Loss of NE integrity was not due simply to an

217 absence of membrane, since all CHMP7-positive micronuclei labelled for the NE

218 marker LAP2, and indeed labeled more strongly for this marker than CHMP7-

219 negative micronuclei or parent nuclei (Figure 4G, H).

221 This finding prompted us to investigate if these micronuclei are 'collapsed' and

222 hence their chromatin core is invaded by NE/ER membrane ${ }^{2}$. Such micronuclear 
envelope collapse is induced by nuclear lamina defects, and this weakness is

associated with catastrophic DNA damage, genomic instability and inflammation 3,14,30,31. Indeed, ER membrane was enriched within the core region of

PDI signal much more intense than over the rest of the cell (Figure 5A-C). In

intensity (Figure 5A, B). In summary, CHMP7 accumulates on micronuclei that have

a disrupted $N E$, and which are infiltrated with ER membrane.

231 Taken together, these data show that ESCRT-III accumulates selectively on

232 micronuclei that lack NE integrity and recapitulate the phenotype occurring at the

233 primary nucleus when VPS4 is depleted. Thus, ESCRT-III activity is impaired at a

234 subset of micronuclei in interphase cells.

236 ESCRT-III accumulates preferentially to acentric micronuclei containing fragmented

237 DNA.

238 ESCRT-III accumulates on a subset of micronuclei. To determine whether these are

239 derived primarily from aneugenic or clastogenic events, we quantified the

240 proportion of ESCRT-III micronuclei containing whole or partial chromosomes, using

241 CREST staining ${ }^{28}$. CHMP7-labelled micronuclei mostly lacked CREST staining (Figure

242 6A, B). In contrast, most CHMP7-negative micronuclei labeled for CREST (Figure 6A, 
B). CHMP7-positive micronuclei were also much smaller than CHMP7-negative

244 micronuclei $(\sim 1.5 \mu \mathrm{m}$ in diameter on average compared to $\sim 2.2 \mu \mathrm{m}$, representing a

$245 \sim 50 \%$ smaller volume) (Figure 6C), consistent with them containing chromosome

246 fragments.

248 UVC irradiation is a clastogenic agent, generating single-stranded DNA lesions that

mitotic partitioning of chromosome fragments into micronuclei, which accumulate

ssDNA-binding proteins. Therefore, to confirm that ESCRT-III accumulates

UVC irradiation to selectively increase this population of micronuclei. HeLa cells

were irradiated with a moderate dose of UVC, and the number and phenotype (i.e.

labeling for $\mathrm{CHMP7}$ and/or the double-strand break marker $\mathrm{YH} 2 \mathrm{AX}$ ) of micronuclei

were analyzed over 72 hours recovery. The number of micronuclei per cell increased

257 after 24 hours and rose $\sim$ 4-fold within 48 hours (Figure 6D, E), a timing indicative of

258 the need for cells to go through mitosis in order to form micronuclei ${ }^{34}$. Aligned with

259 this increase, the proportion of micronuclei that were CHMP7-positive also rose

260 significantly (Figure 6F). As expected, labeling of micronuclei for $\mathrm{YH} 2 \mathrm{AX}$ also

261 increased, though here the pattern was more complex. The population of $\mathrm{yH} 2 \mathrm{AX}$ -

262 positive micronuclei first increased modestly around $1 \mathrm{hr}$ post-treatment,

263 highlighting a minor population of pre-existing micronuclei that are directly subject 
264 to DNA damage (Figure 6G). A second, larger increase in the proportion of

265 micronuclei labeled for $\mathrm{YH} 2 \mathrm{AX}$, and for both CHMP7 and $\mathrm{YH} 2 \mathrm{AX}$, was apparent

over 24-48 hours (Figure 6G,H).

267 In summary, acentric micronuclei are preferentially enriched by ESCRT-III; consistent

268 with this, clastogenic agents such as UV21,22,30,35,36 increase the population of

269 micronuclei that contain ESCRT-accumulations.

271 CHMP7 is important for generating ssDNA and retaining cGAS to a pool of

272 micronuclei.

273 Collectively, our data point to a pool of ESCRT-III that preferentially accumulates on

274 micronuclei lacking NE integrity and compartmentalization. However, ESCRT-III is not the primary cause of these defects in micronuclear organization, since they are more frequent when ESCRT-III function is lacking. Since damage to micronuclei can generate a source of immunostimulatory DNA ${ }^{14}$, we next examined the role of

278 ESCRT-III in this downstream process. Micronuclei enriched for CHMP4B 279 preferentially stained strongly for replication protein A (RPA), a protein that binds 280 directly to ssDNA (Figure 7A,B). Similarly, cGAS, is known to enter ruptured 281 micronuclei to bind exposed DNA and indeed was concentrated in nearly all those

282 accumulating CHMP7 (Figure 7C,D). Significantly, CHMP7 depletion reduced the 283 proportion of micronuclei that were enriched for RPA (Figure 7E,F) or cGAS (Figure 
284 7G,H). In contrast, VPS4KD did not appreciably affect RPA or cGAS enrichment at

285 micronuclei (Figure 7E-H), despite its negative impact on micronuclear integrity

286 (Supplemental Figure 2B and Figure 2H).

287 In summary, the co-enrichment of ESCRT-III and RPA/cGAS at a subset of

288 micronuclei and the absence of RPA/cGAS enrichment upon CHMP7 depletion,

289 point to a non-canonical (VPS4-independent) function for ESCRT-III in maintaining a

290 pool of cytosolic DNA in cancer cells.

292 4. Discussion

293 We have addressed how the ESCRT-III membrane-repair complex supports genomic

294 stability via its effects on aberrant structures associated with chromosomal instability

295 and genotoxic events, namely micronuclei. Micronuclear collapse contributes to the

296 accumulation of damaged DNA arising as a result of NE rupture, increasing the

297 likelihood of failed DNA replication and persistent genomic instability. Therefore,

298 the existence of a mechanism for protecting the DNA within micronuclei is

299 plausible, in order to avoid chromosome shattering and chromothripsis $3,37$.

300 Whilst the process of reformation of a micronuclear envelope is not well

301 understood, almost 100\% of spontaneously arising micronuclei display successful

302 nucleocytoplasmic compartmentalisation upon exit from mitosis ${ }^{2}$. These micronuclei

303 must have a sealed NE, a process carried out at the primary nucleus by the 
304 CHMP7/ESCRT-III/VPS4 system ${ }^{17}$. Indeed, ESCRT-III dynamics at lagging

305 chromosomes during telophase appears normal ${ }^{38}$. We show here that loss of

306 CHMP7 or VPS4 leads to increased occurrence of micronuclei with a ruptured and

307 collapsed NE, supporting a role for ESCRT-III in sealing micronuclear envelopes

308 (VPS4-dependent pathway, Supplemental Figure 4A).

309 Importantly, however, our data also highlight a population of ESCRT-III that

310 apparently is not subject to rapid, VPS4-dependent turnover, which accumulates

311 within micronuclei (Supplemental Figure 4A). Two questions arise: what mechanisms

312 lead to the generation of this 'persistent' pool of ESCRT-III, and does the pool have

313 a selective role at micronuclei that is distinct from membrane repair?

314 To identify events that lead to ESCRT-III accumulations on micronuclei, we have

315 comprehensively characterized the nature of these structures. Most have a broken

316 lamina and are infiltrated by ER membranes, with a consequent loss of

317 compartmentalization. These phenotypes are more rarely observed in micronuclei

318 that lack CHMP7. Such defects might be due to aberrant micronuclear NE assembly

319 after mitosis ${ }^{5}$ and/or failure to repair a ruptured micronuclear NE during interphase ${ }^{2}$.

320 At the primary nucleus, the recruitment of ESCRT-III is so transient ${ }^{7,10}$ that depletion

321 of VPS4 is necessary to slow down ESCRT-III turnover at the membrane. Our

322 VPS4KD data imply the existence of a similar mechanism (VPS4-dependent

323 pathway, Supplemental Figure 4A); hence, the accumulation of CHMP7 and 
324 CHMP4B observed in unperturbed cancer cells implies the normal balance between

325 ESCRT-III assembly and disassembly is impaired and/or is not regulated by VPS4

326 (VPS4-independent pathway, Supplemental Figure 4A).

327 ESCRT-III-enriched micronuclei are smaller than that lacking ESCRT-III and are

328 predominantly acentric; they therefore most likely contain chromosome fragments,

329 such as those generated by DNA damage in previous cell cycles ${ }^{39}$. Hence, ESCRT-III

330 accumulation might result from aberrant NE formation around chromosomal

331 fragments, in which the NE membrane is subject to an unusually high degree of

332 curvature and is prone to rupture ${ }^{40}$. One potential explanation for impaired ESCRT-

333 III turnover at acentric micronuclei could be the lack of a regulatory activity that

334 relies on signalling from the centromere.

335 Steric confinement provided by dense spindle microtubules at telophase causes

336 uneven loading of nuclear envelope proteins on lagging chromosomes,

337 consequently generating micronuclei with a defective nuclear envelope ${ }^{38}$. This

338 phenomenon explains why such micronuclei may spontaneously rupture later in

339 interphase; therefore, it is possible that ESCRT-III is recruited to micronuclei arising

340 from this pathway.

341 Is there a role for aberrant ESCRT-III accumulations? ESCRT-III enrichment at

342 micronuclei may cause further damage to DNA (Supplemental Figure 4A), as

343 suggested by the significant association between ESCRT-III and RPA, which marks 
344 the presence of single stranded DNA ${ }^{41}$. Both RPA and cGAS enrichment in

345 micronuclei are lost by depleting CHMP7 but not VPS4 (Figure 7), arguing that the

346 accumulation of ESCRT-III is required to generate higher levels of damaged DNA

347 (Supplemental Figure 4B).

349 The implications for aberrant NE remodelling by ESCRT-III at micronuclei may be far

350 further reaching than simply explaining a genome predisposition to aneuploidy ${ }^{19,42}$

or chromothripsis caused by loss of nuclear envelope repair activity.

352 Ruptured micronuclei are key signals to activate immune pathways that can control tumor progression ${ }^{14,43}$ via the cGAS-STING (STimulator of Interferon Gene)

354 pathway $^{14}$. cGAS recognition drives immune responses, senescence ${ }^{44,45}$ and the

355 elimination of cytosolic DNA by autophagy ${ }^{46}$. The ESCRT-III subunit CHMP4B was

356 previously localized at micronuclei and a link with autophagic degradation of

357 cytosolic DNA was suggested ${ }^{47}$.

358 CHMP7 depletion decreases the population of cGAS-enriched micronuclei. The observed reduction is similar in magnitude to the proportion of micronuclei with

360 ESCRT-III accumulations in unperturbed cells ( 20\%). This suggests that ESCRT-III supports cGAS in ruptured micronuclei, perhaps by enhancing the production of

362 DNA structures recognized by $\mathrm{CGAS}^{14,16}$. It is unlikely that CHMP7 depletion simply

363 creates unstable micronuclei that lose cGAS, as VPS4 depletion does not reduce 
364 either the pool of RPA or cGAS-enriched micronuclei even though VPS4-depleted

366 In the context of cancer progression, short lived cGAS signaling may be beneficial

367 to induce antitumor responses; conversely, it may contribute to cancer progression

368 if persistently sustained ${ }^{48}$. Higher CHMP7 and cGAS ${ }^{44}$ RNA expression correlate with

369 higher frequency of patient survival in lung adenocarcinoma (Supplemental Figure

370 4C, D), a tumor with high levels of chromosomal instability ${ }^{2,48}$. Moreover, CHMP7

371 has been identified as gene that negatively-regulates cell proliferation (STOP gene),

372 in a study showing how recurrent deletions occurring in cancers drive

373 tumorigenesis ${ }^{49}$. The CHMP7 gene is located on the short arm of chromosome 8

374 (8p21.3). Chromosomal deletions on this region are common in carcinomas ${ }^{50-54}$ and

375 loss of heterozygosity in $8 p$ regulates tumour progression and drug resistance ${ }^{55}$.

376 Therefore, it is intriguing to speculate that among the functions of ESCRT-III there is

377 a role for aberrant membrane remodeling activity that damages DNA, expose it to

378 the cytosol, thus promoting the activation of pro-inflammatory pathways that

379 modulate the tumor microenvironment.

\section{5. Methods}

382 Cell lines, transfections and sRNAi knockdowns. HeLa, HeLaM, U2OS and h-TERT 
384 bovine serum and $1 \%$ non-essential amino acids (Lonza). Cells were mycoplasma-

385 free, with regular checks performed using the Mycoalert Mycoplasma Detection Kit

386 (Lonza).

387 Depletion of target proteins was carried out using DHARMAFECT and ON-

388 TARGETplus sRNAis per manufacturer's instructions (Thermo Scientific: Dharmacon

389 Products, Lafayette, CO, USA) (see Supplementary Table 1 for sRNAi sequences).

390 Cells were plated 24 hours prior to knockdown, transfected once with sRNAi for 24-

39172 hours at the appropriate concentration (usually $20 \mathrm{nM}$ otherwise stated)

392 dissolved in serum-free DMEM. The efficiency of sRNAi depletion was assessed by

393 immunoblotting or quantitative PCR.

394 To visualize GFP-NLS in CHMP7 knockdown experiments, HeLa and h-TERT-

395 immortalized Human Fibroblast (HF) cells were transfected with sRNAi CHMP7

$39620 \mathrm{nM}^{19}$ or control sRNAi (GLi2 20nM) ${ }^{56} 24$ hours after seeding, then transfected with

$3971 \mu \mathrm{g}$ pEGFP-NLS(SV40) (Kind gift from Dr. Patrizia Lavia) and fixed at 48 hours post-

398 transfection.

400 Quantitative PCR. Quantitative PCR was used to assess VPS4A depletion in HeLa

401 cells $^{57}$. Briefly, RNA was purified from cell pellets using the Absolutely RNA

402 Microprep kit (Stratagene). First strand cDNA was synthesized using random primers

403 with the Multiple Temperature cDNA Synthesis Kit (Stratagene). Samples were 
404 stored at $-20^{\circ} \mathrm{C}$. RT-qPCR was performed using the SYBR-GREEN JumpStart Taq

405 Ready Mix (Sigma). Conditions used were: initial denaturation at $94^{\circ} \mathrm{C}$ for 2 minutes,

406 followed by 40 cycles of denaturation at $94^{\circ} \mathrm{C}$ for 15 seconds, annealing at $53.7^{\circ} \mathrm{C}$

407 for $1 \mathrm{~min}, 72^{\circ} \mathrm{C}$ for $1 \mathrm{~min}$. Tata Binding Protein (TBP) was used as a loading control

408 for the qPCR.

409 TBP amplification primers: forward, 5'-GCCCGAAACGCCGAATATAATCCC-3';

410 reverse, 5'-GGACTGTTCTTCACTCTTGGCTCCTG-3'. VPS4A primers: forward, 5'-

411 GACAACGTCAACCCTCCAG-3'; reverse, 5'-AGCGCCTCCTCGTAGTTCTT-3'.

412 Depletion of VPS4A was at least $90 \%$ effective as judged by RT-PCR.

413

414 Immunofluorescence microscopy. Cells were fixed with 4\% paraformaldehyde (PFA)

415 for 15 minutes at room temperature and permeabilized with $0.5 \%$ Triton $\mathrm{X}-100$ for

41620 minutes. Primary (Supplementary Table 2) and secondary antibodies were

417 incubated in 1\% BSA in in PBS containing $0.1 \%$ Triton X-100 in a humidified

418 chamber at $4^{\circ} \mathrm{C}$. Coverslips were mounted with VECTASHIELD (Vector Laboratories)

419 or ProLong Gold (Invitrogen) mounting medium containing $1.5 \mu \mathrm{g} / \mathrm{ml}$ DAPI.

420 Fluorescence was detected using a Nikon Eclipse TE200 microscope with 60x or

421 100x objectives. Images were acquired using a Hamamatsu C4742-96-12G04 digital

422 CCD camera and Volocity imaging software (Perkin Elmer), and analyzed using $423 \mathrm{FiJi}{ }^{58}$. 
424 An Applied Precision Deltavision deconvolution microscope with a UplanSApo 100x

430 STED microscopy. STED images of cells stained with To-Pro3 (Thermofisher) or

431 Abberior STAR- (Abberior GmbH, Germany), Alexa Flour- or Oregon Green-

432 (ThermoFisher Scientific, Australia) conjugated secondary antibodies diluted 1:300 were acquired with a Leica TCS SP8 microscope (Leica Microsystems GmbH,

434 Mannheim, Germany) using a Leica HCPL Apo 100×/1.40 oil STED white objective, and equipped for 3D-gated- STED microscopy with a white light laser (tunable from

$436470 \mathrm{~nm}$ to $670 \mathrm{~nm}$ ) for excitation, $775 \mathrm{~nm}$ and $592 \mathrm{~nm}$ lasers for STED depletion

437 and Hybrid Detectors. STED lasers were operated at 50\% output power. Emitted

438 fluorescence was filtered using notch filters (775 nm, 592 or $488 \mathrm{~nm}$ ). Images were

439 acquired using sequential scanning with a line average of 2, a frame accumulation

440 of 3 and a scan speed of $600 \mathrm{~Hz}$. Images contained $1024 \times 1024$ pixels. Pixel size was

$44125 \mathrm{~nm}(X, Y)$ and $67 \mathrm{~nm}(Z)$. Each z series (with sections at $67 \mathrm{~nm}$ intervals) was

442 acquired using Leica Application Suite (LAS), then deconvolved using Huygens

443 Professional software (Scientific Volume Imaging, The Netherlands). Images were 444 analyzed using LAS and FiJi ${ }^{58}$. 
446 Micronuclei scoring. Micronuclei from random fields of view in unsynchronized,

447 untreated or sRNAi-treated cells were scored for enrichment in CHMP7 or CHMP4B

448 and for a second marker.Only interphase, non-apoptotic cells were analyzed (cells

449 containing $>3$ micronuclei were excluded to further minimize the possibility of

450 including apoptotic cells). Micronuclei were defined by criteria based on Fenech et

451 al. ${ }^{59}$ : their diameter should not exceed $1 / 3$ that of the primary nucleus; they should

452 be separated from the primary nucleus; they should have similar intensity of DAPI

453 staining to the primary nucleus but occasionally may stain more intensively.

454 Micronuclei were identified first by DAPI staining and were then scored for the

455 presence or absence of $\mathrm{CHMP7}$, appearing as an intense pan-micronuclear signal or

456 large foci. Other phenotypes were quantified based on visual assessment of various

457 markers, with fluorescence intensity over the primary nucleus taken as a reference 458 where appropriate.

460 Statistical analysis of micronuclei categories. Data were analysed as in Hatch et $\mathrm{al}^{2}$.

461 Briefly, the statistical difference between two or more categories of micronuclei was

462 determined using Fisher's exact test (for a $2 \times 2$ table) or $\chi 2$ analysis on the raw

463 counts to obtain a p-value. The Null hypothesis assumed was that all phenotypes

464 occurred with the same frequency. The Cramer $V$ coefficient (or $\phi$ coefficient for a 2 
$465 \times 2$ table) was used to assess the strength of association between variables. The

466 difference between independent replicates was determined by comparing p-values

467 for each individual experiment. Raw counts were pooled and a final $p$ value

468 calculated if the p-values of individual replicates resulted in statistical significance.

\section{Competing interests}

471 We have no competing interests.

472

\section{$473 \quad 7$. Acknowledgements}

474 BC and HB acknowledge the University of Sheffield for a PhD studentship to JW

475 and the EPSRC for a studentship to AJC. BC acknowledges the EPSRC grant

476 EP/M027821/1. PGW acknowledges the BBSRC grant BB/M000877/1. NFR

477 acknowledges funding from the Children's Medical Research Institute. CR

478 acknowledges the AIRC grant IG\#17739. The authors acknowledge the facilities and

479 the scientific and technical assistance of the Australian Microscopy \& Microanalysis

480 Research Facility at the Australian Centre for Microscopy \& Microanalysis at the

481 University of Sydney and the Light Microscopy Facility at the University of Sheffield. 


\section{References}

1 Fenech M, Kirsch-Volders M, Natarajan AT, Surralles J, Crott JW, Parry J et al.

484 Molecular mechanisms of micronucleus, nucleoplasmic bridge and nuclear bud formation in mammalian and human cells. Mutagenesis 2010; 26: 125-132.

4862 Hatch EM, Fischer AH, Deerinck TJ, Hetzer MW. Catastrophic nuclear envelope collapse in cancer cell micronuclei. Cell 2013; 154: 47-60.

489 Chromothripsis from DNA damage in micronuclei. Nature 2015; 522: 179-184.

4904 Terradas M, Martín M, Genescà A. Impaired nuclear functions in micronuclei results in genome instability and chromothripsis. Archives of Toxicology 2016; 90: 2657-2667.

5 de Castro IJ, Gil RS, Ligammari L, Di Giacinto ML, Vagnarelli P. CDK1 and PLK1 coordinate the disassembly and reassembly of the nuclear envelope in vertebrate mitosis. Oncotarget 2018; 9: 7763-7773.

6 Terradas M, Martín M, Hernández L, Tusell L, Genescà A. Nuclear envelope defects impede a proper response to micronuclear DNA lesions. Mutation Research/Fundamental and Molecular Mechanisms of Mutagenesis 2012; 729: 35-40.

7 Raab M, Gentili M, de Belly H, Thiam HR, Vargas P, Jimenez AJ et al. ESCRT III repairs nuclear envelope ruptures during cell migration to limit DNA damage and cell death. Science 2016; 352: 359-362.

8 Robijns J, Molenberghs F, Sieprath T, Corne TDJ, Verschuuren M, De Vos WH. In silico synchronization reveals regulators of nuclear ruptures in lamin A/C deficient model cells. Sci Rep 2016; 6: 30325.

5069 Denais CM, Gilbert RM, Isermann P, McGregor AL, Lindert te M, Weigelin B et 507 al. Nuclear envelope rupture and repair during cancer cell migration. Science 2016; 352: 353-358.

10 Olmos Y, Hodgson L, Mantell J, Verkade P, Carlton JG. ESCRT-III controls nuclear envelope reformation. Nature 2015; 522: 236-239.

51111 Olmos Y, Perdrix-Rosell A, Carlton JG. Membrane Binding by CHMP7 
12 Gu M, Lajoie D, Chen OS, Appen von A, Ladinsky MS, Redd MJ et al. LEM2 recruits CHMP7 for ESCRT-mediated nuclear envelope closure in fission yeast and human cells. Proc Natl Acad Sci USA 2017; : 201613916.

13 Henne WM, Stenmark H, Emr SD. Molecular mechanisms of the membrane sculpting ESCRT pathway. Cold Spring Harbor Perspectives in Biology 2013; 5. doi:10.1101/cshperspect.a016766.

14 Mackenzie KJ, Carroll P, Martin C-A, Murina O, Fluteau A, Simpson DJ et al. cGAS surveillance of micronuclei links genome instability to innate immunity. Nature 2017; 548: 461-465.

15 Chen Q, Sun L, Chen ZJ. Regulation and function of the cGAS-STING pathway of cytosolic DNA sensing. Nat Immunol 2016; 17: 1142-1149.

16 Civril F, Deimling T, de Oliveira Mann CC, Ablasser A, Moldt M, Witte G et al. Structural mechanism of cytosolic DNA sensing by cGAS. Nature 2013; 498: 332-337.

17 Vietri M, Stenmark H, Campsteijn C. Closing a gap in the nuclear envelope. Current Opinion in Cell Biology 2016; 40: 90-97.

18 Vietri M, Schink KO, Campsteijn C, Wegner CS, Schultz SW, Christ L et al. Spastin and ESCRT-III coordinate mitotic spindle disassembly and nuclear envelope sealing. Nature 2015; 522: 231-235.

19 Morita E, Colf LA, Karren MA, Sandrin V, Rodesch CK, Sundquist WI. Human ESCRT-III and VPS4 proteins are required for centrosome and spindle maintenance. Proc Natl Acad Sci USA 2010; 107: 12889-12894.

20 De Vos WH, Houben F, Kamps M, Malhas A, Verheyen F, Cox J et al. Repetitive disruptions of the nuclear envelope invoke temporary loss of cellular compartmentalization in laminopathies. Hum Mol Genet 2011; 20: 4175-4186.

21 Xu B, Sun Z, Liu Z, Guo H, Liu Q, Jiang H et al. Replication Stress Induces Micronuclei Comprising of Aggregated DNA Double-Strand Breaks. 2011; 6: e18618-11.

22 Ward IM, Chen J. Histone H2AX is phosphorylated in an ATR-dependent manner in response to replicational stress. J Biol Chem 2001; 276: 4775947762.

23 Wolf C, Rapp A, Berndt N, Staroske W, Schuster M, Dobrick-Mattheuer M et al. RPA and Rad51 constitute a cell intrinsic mechanism to protect the cytosol from self DNA. Nature Communications 2016; 7: 11752. 
24 Shimizu N. Molecular mechanisms of the origin of micronuclei from extrachromosomal elements. 2010; 26: 119-123.

55025 Utani K-I, Okamoto A, Shimizu N. Generation of Micronuclei during Interphase by Coupling between Cytoplasmic Membrane Blebbing and Nuclear Budding. PLOS ONE 2011; 6: e27233-12.

26 Thoresen SB, Campsteijn C, Vietri M, Schink KO, Liestøl K, Andersen JS et al. ANCHR mediates Aurora-B-dependent abscission checkpoint control through retention of VPS4. Nat Cell Biol 2014; 16: 550-560.

27 Agromayor M, Martin-Serrano J. Knowing when to cut and run: mechanisms that control cytokinetic abscission. Trends in Cell Biology 2013. doi:10.1016/j.tcb.2013.04.006.

28 Norppa H, Falck GC-M. What do human micronuclei contain? Mutagenesis

29 Bakhoum SF, Silkworth WT, Nardi IK, Nicholson JM, Compton DA, Cimini D. The mitotic origin of chromosomal instability. Current Biology 2014; 24: R148R149.

30 Shah P, Wolf K, Lammerding J. Bursting the Bubble - Nuclear Envelope Rupture as a Path to Genomic Instability? Trends in Cell Biology 2017; : 1-10.

31 Gekara NO. DNA damage-induced immune response: Micronuclei provide key platform. J Cell Biol 2017; 216: 2999-3001.

32 Marti TM, Hefner E, Feeney L, Natale V, Cleaver JE. H2AX phosphorylation within the G1 phase after UV irradiation depends on nucleotide excision repair and not DNA double-strand breaks. Proc Natl Acad Sci USA 2006; 103: 98919896.

33 Gabrielli BG, Clark JM, McCormack AK, Ellem KA. Ultraviolet light-induced G2 phase cell cycle checkpoint blocks cdc25-dependent progression into mitosis. Oncogene 1997; 15: 749-758.

34 Harding SM, Benci JL, Irianto J, Discher DE, Minn AJ, Greenberg RA. Mitotic progression following DNA damage enables pattern recognition within micronuclei. Nature 2017; 548: 466-470.

57835 Gelot C, Magdalou I, Lopez B. Replication Stress in Mammalian Cells and Its Consequences for Mitosis. Genes 2015; 6: 267-298. 
36 Sabatinos SA, Ranatunga NS, Yuan J-P, Green MD, Forsburg SL. Replication stress in early $\mathrm{S}$ phase generates apparent micronuclei and chromosome rearrangement in fission yeast. Mol Biol Cell 2015; 26: 3439-3450.

583

584

585

586

587

588

589

590

591

592

593

594

595

596

597

598

599

600

601

602

603

604

605

606

607

608

609

610

611

37 Ly P, Teitz LS, Kim DH, Shoshani O, Skaletsky H, Fachinetti D et al. Selective Y centromere inactivation triggers chromosome shattering in micronuclei and repair by non-homologous end joining. Nat Cell Biol 2017; 19: 68-75.

38 Liu S, Kwon M, Mannino M, Yang N, Renda F, Khodjakov A et al. Nuclear envelope assembly defects link mitotic errors to chromothripsis. Nature 2018; 561: 551-555.

39 Crasta K, Ganem NJ, Dagher R, Lantermann AB, Ivanova EV, Pan Y et al. DNA breaks and chromosome pulverization from errors in mitosis. Nature 2012; 482: 53-58.

40 Xia Y, Ivanovska IL, Zhu K, Smith L, Irianto J, Pfeifer CR et al. Nuclear rupture at sites of high curvature compromises retention of DNA repair factors. J Cell Biol 2018; 217: 3796-3808.

41 Haaf T, Raderschall E, Reddy G, Ward DC, Radding CM, Golub El. Sequestration of mammalian Rad51-recombination protein into micronuclei. $J$ Cell Biol 1999; 144: 11-20.

42 Carlton JG, Caballe A, Agromayor M, Kloc M, Martin-Serrano J. ESCRT-III governs the Aurora B-mediated abscission checkpoint through CHMP4C. Science 2012; 336: 220-225.

43 Bakhoum SF, Ngo B, Laughney AM, Cavallo J-A, Murphy CJ, Ly P et al. Chromosomal instability drives metastasis through a cytosolic DNA response. Nature 2018; 553: 467-472.

44 Yang $\mathrm{H}$, Wang $\mathrm{H}$, Ren J, Chen Q, Chen ZJ. cGAS is essential for cellular senescence. Proc Natl Acad Sci USA 2017; 114: E4612-E4620.

45 Glück S, Guey B, Gulen MF, Wolter K, Kang T-W, Schmacke NA et al. Innate immune sensing of cytosolic chromatin fragments through cGAS promotes senescence. Nat Cell Biol 2017; : 1-25.

46 Bartsch K, Knittler K, Borowski C, Rudnik S, Damme M, Aden K et al. Absence of RNase $\mathrm{H} 2$ triggers generation of immunogenic micronuclei removed by autophagy. Hum Mol Genet 2017; 26: 3960-3972. 
47 Sagona AP, Nezis IP, Stenmark H. Association of CHMP4B and autophagy with micronuclei: implications for cataract formation. Biomed Res Int 2014; 2014: 974393-10.

48 Bakhoum SF, Cantley LC. The Multifaceted Role of Chromosomal Instability in Cancer and Its Microenvironment. Cell 2018; 174: 1347-1360.

49 Solimini NL, Xu Q, Mermel CH, Liang AC, Schlabach MR, Luo J et al. Recurrent hemizygous deletions in cancers may optimize proliferative potential. Science 2012; 337: 104-109.

50 Emi M, Fujiwara Y, Nakajima T, Tsuchiya E, Tsuda H, Hirohashi S et al. Frequent loss of heterozygosity for loci on chromosome $8 p$ in hepatocellular carcinoma, colorectal cancer, and lung cancer. Cancer Res 1992; 52: 5368-5372.

51 Yaremko ML, Kutza C, Lyzak J, Mick R, Recant WM, Westbrook CA. Loss of heterozygosity from the short arm of chromosome 8 is associated with invasive behavior in breast cancer. Genes, Chromosomes and Cancer 1996; 16: 189195.

52 Kang J. Genomic alterations on 8p21-p23 are the most frequent genetic events in stage I squamous cell carcinoma of the lung. Exp Ther Med 2015; 9: 345350.

53 Takle LA, Knowles MA. Deletion mapping implicates two tumor suppressor genes on chromosome $8 p$ in the development of bladder cancer. Oncogene 1996; 12: 1083-1087.

54 Pineau P, Nagai H, Prigent S, Wei Y, Gyapay G, Weissenbach J et al. Identification of three distinct regions of allelic deletions on the short arm of chromosome 8 in hepatocellular carcinoma. Oncogene 1999; 18: 3127-3134.

55 Cai Y, Crowther J, Pastor T, Abbasi Asbagh L, Baietti MF, De Troyer M et al. Loss of Chromosome 8p Governs Tumor Progression and Drug Response by Altering Lipid Metabolism. Cancer Cell 2016; 29: 751-766.

56 Barbiero I, Valente D, Chandola C, Magi F, Bergo A, Monteonofrio L et al. CDKL5 localizes at the centrosome and midbody and is required for faithful cell division. Sci Rep 2017; 7: 6228.

57 Stefani F, Zhang L, Taylor S, Donovan J, Rollinson S, Doyotte A et al. UBAP1 is a component of an endosome-specific ESCRT-I complex that is essential for MVB sorting. Curr Biol 2011; 21: 1245-1250. 
bioRxiv preprint doi: https://doi.org/10.1101/476630; this version posted December 1, 2018. The copyright holder for this preprint (which

was not certified by peer review) is the author/funder, who has granted bioRxiv a license to display the preprint in perpetuity. It is made available under aCC-BY-NC-ND 4.0 International license.

64558 Schindelin J, Arganda-Carreras I, Frise E, Kaynig V, Longair M, Pietzsch T et al. 646 Fiji: an open-source platform for biological-image analysis. Nature Methods $647 \quad 2012$ 9:7 2012; 9: 676-682.

64859 Fenech M. Cytokinesis-block micronucleus cytome assay. Nat Protoc 2007; 2: 649 1084-1104.

650 


\section{Figure legends}

652 Figure 1. CHMP7 and VPS4 depletion cause general nuclear envelope

653 disorganisation. (A) Examples of the honeycomb and herniated Lamin A and B 654 phenotypes in HeLa cells treated with CHMP7 sRNAi for 48 hours. Arrowheads

655 indicate chromatin protruding out the nucleus in herniated cells. (B) Quantification

656 of Lamin A and B defects in Hela cells treated with the indicated sRNAi (minimum of

657300 cells scored per condition). (C) Examples of CHMP7 foci in HeLa cells treated 658 with VPS4 and NT-control sRNAi for 48 hours and associated with LAP2 or Lamin A 659 foci (LAP2 and LaminA enriched, arrowheads) or associated with Lamin B holes 660 (HeLaM). (D) Average CHMP7 foci number per nucleus in HeLa ( $n=305)$, HeLa M $661 \quad(n=222)$ and U2OS $(n=257)$ cells treated with VPS4 sRNAi for 48 hours. (E)

662 Quantification of CHMP7 foci in relation to LAP2, Lamin A/C or Lamin B, in HeLa 663 cells treated with VPS4 sRNAi for 48 hours (minimum of 400 foci for each condition).

664 (F) Deconvolved widefield image of CHMP4B co-localisation with CHMP7 at nuclear 665 envelope foci in HeLa cells transfected with VPS4 sRNAi for 48 hours (top panel) and 666 CHMP7/CHMP4B distribution in control cells (bottom panel). (G) Percentage of 667 HeLa cells containing at least one large accumulation of CHMP7 (focus) following 668 VPS4 sRNAi treatment at 24, 48 and 72 hours post transfection (minimum of 200 669 nuclei scored for each condition). (H) Number of nuclear CHMP foci accumulating in 670 HeLa M cells under the indicated sRNAi treatments. (minimum of 100 nuclei scored 
671 for each condition). Averages and SEM are shown ( $=3$ ). Scale bars represent $67210 \mu \mathrm{m}$.

673 Figure 2. Impairment of ESCRT-III function affects micronuclei integrity. (A) HeLa

674 cells were transfected with the indicated sRNAi for $48 \mathrm{~h}$ and the percentages of cells

675 with at least one micronucleus were quantified for each treatment (minimum 250

676 cells per treatment, per repeat). Results were analyzed using a one-way ANOVA

677 with Dunnett's post hoc test. (B) HeLa cells were transfected with the indicated

678 sRNAi for either 24, 48 or 72 hours. The number of micronucleated cells were

679 quantified for each one of the treatments (minimum 200 cells were scored for each

680 treatment, per repeat). Results were analyzed using a two-way ANOVA with

681 Dunnett's post hoc test. (C) The average number of micronuclei observed per

682 micronucleated cell were quantified (minimum 150 cells per treatment, per repeat).

683 Results were analyzed using a one-way ANOVA with Dunnett's post hoc test. (D)

684 Micronuclei in HeLa cells transfected for 48h with the indicated sRNAi and scored

685 for presence of CREST (minimum 200 micronuclei per treatment, per repeat).

686 Results were analyzed using a one-way ANOVA with Dunnett's post hoc test. (E)

687 HeLa cells were transfected with the indicated sRNAi for 48 hours and scored for

688 the status of Lamin B, as defined in supplementary Figure 2A (minimum 100

689 micronuclei per treatment, per repeat). Results were analyzed using a one-way

690 ANOVA with Dunnett's post hoc test. Statistics shown for the 'absent' category. (F)

691 HeLa cells were transfected with the indicated sRNAi for 48 hours and scored for 
692 the status of mab414, as defined in supplementary Figure 2B (minimum 100

693 micronuclei per treatment, per repeat). Results were analyzed using a one-way

694 ANOVA with Dunnett's post hoc test. Statistics shown for the 'absent' category. (G)

695 Micronuclei in HeLa cells transfected for 48h with the indicated sRNAi and scored

696 for presence of PDI within micronuclear chromatin (PDI-invaded; minimum 100

697 micronuclei per treatment, per repeat). Results were analyzed using a one-way

698 ANOVA with Dunnett's post hoc test. $(\mathrm{H})$ Micronuclei in HeLa cells transfected for

699 48h with the indicated sRNAi and scored for presence of PARP1 (minimum 100

700 micronuclei per treatment, per repeat). Results were analyzed using a one-way

701 ANOVA with Dunnett's post hoc test. Averages and SEM are shown $(\mathrm{N}=3$, unless

702 stated otherwise). (I) HeLa cells treated with control or CHMP7 sRNAi, then

703 transfected with GFP-NLS post-seeding and imaged 48 hours post sRNAi depletion.

704 Examples of micronuclei with GFP-NLS absent (top panel) or present (bottom panel)

705 (arrowheads). Scale bar $10 \mu \mathrm{m}$. (J) Quantification of GFP-NLS retention in

706 micronuclei treated as in (G) as judged visually by the loss of nuclear signal (25

707 micronuclei scored per treatment, per repeat). Averages of $\mathrm{N}=2$ biological repeats

708 are shown.

709 Figure 3. ESCRT-III accumulates on micronuclei and is recruited by CHMP7. (A)

710 Untreated HeLa cells showing accumulations of CHMP7 and CHMP4B at a

711 micronucleus (arrowhead). Scale bars 10um. (B) U2OS, HeLa and HeLa M cell lines

712 were transfected with non-targeting or VPS4A and B sRNAi for 48h, and stained for 
713 CHMP7. Micronuclei were scored for presence or absence of CHMP7 (minimum of

714400 micronuclei scored per treatment). (C) Percentage of micronuclei in HeLa cells

715 transfected with the indicated sRNAi for 48 hours displaying enriched CHMP7 or

716 CHMP4 (minimum 200 micronuclei scored per treatment). (D) Confocal microscopy

717 in STED mode of a micronucleus in a control HeLa cell containing CHMP7 and

718 CHMP4B accumulations. Scale bars represent $1 \mu \mathrm{m}$. (E) Confocal microscopy in

719 STED mode of the micronucleus in (D) showing z-slicing across $335 \mathrm{~nm}$ starting from

720 the top going towards the inside of the micronucleus. Scale bar $1 \mu \mathrm{m}$. Averages and

721 SEM are shown $(\mathrm{N}=3)$.

722 Figure 4. Micronuclei with CHMP7 accumulations are enriched with LAP2 and have

723 lost nuclear lamina integrity and compartmentalization. (A) Immunofluorescence

724 image of a micronucleus where CHMP7 localises to regions with gaps in Lamin B in

725 control cells (top panel). Examples of CHMP7-positive (arrows) and negative

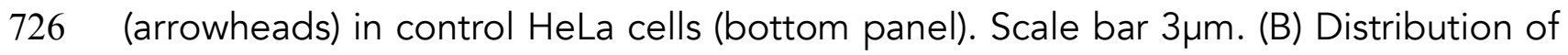

727 the status of CHMP7 and Lamin B in micronuclei (minimum 100 micronuclei scored

728 per repeat). (C) Examples of CHMP7-positive and negative micronuclei with NPC

729 staining in control HeLa cells. (D) Micronuclei in HeLa cells were scored for the

730 status of CHMP7 and the status of NPC (mab414) within the same micronucleus

731 (minimum 160 micronuclei scored per repeat). (E) Examples of CHMP7-positive and

732 negative micronuclei showing absence or presence of PARP1 staining. Scale bar

$7333 \mu \mathrm{m}$. (F) Quantification of the presence or absence of PARP1 and CHMP7 staining 
734 within the same micronucleus (minimum 200 micronuclei scored per repeat). (G)

735 Examples of CHMP7-positive and negative micronuclei with LAP2 staining. Scale

736 bar $3 \mu \mathrm{m}$. $(\mathrm{H})$ Quantification of the presence or absence of LAP2 and CHMP7

737 staining within the same micronucleus (300 micronuclei per repeat). Data were analysed using Fishers' exact test on pooled raw counts distributions $(N=3)$.

Figure 5. Micronuclei with ESCRT-III accumulations display ER membrane invasion.

740

(A) Examples of CHMP7-positive and negative micronuclei with PDI staining. PDI-

excluded micronuclei have endoplasmic reticulum (ER) membrane surrounding their

744 (arrowheads). (B) Distribution of the status of CHMP7 and the presence or absence

745 of PDI invasion within the same micronucleus (minimum 100 micronuclei scored per

746 repeat). Data were analysed using Fishers' exact test on pooled raw counts

747 distributions $(\mathrm{N}=3)$. (C) Example image of a micronucleus in a Hela cell after

748 treatment with VPS4 sRNAi for 48 hours. Accumulation of PDI and CHMP7 within

749 the micronucleus and the irregular shape display membrane infiltration (arrowhead).

750 Scale bars $3 \mu \mathrm{m}$.

751 Figure 6. ESCRT-III preferentially accumulates on acentric micronuclei. (A) HeLa cells

752 transfected with either control or VPS4 sRNAi for 48h hours and co-stained for

753 CREST to detect centromeres, CHMP7 and DAPI. Examples of CHMP7+ve

754 micronuclei that are CREST+ve (arrowhead indicating weak CREST signal) or 

$(p<0.05)$. (C) Control HeLa cells were stained for CHMP7 and DAPI. The diameter of micronuclei measured in ImageJ (75 CHMP7-positive and 75 CHMP7-negative micronuclei). HeLa cells were transfected with sRNAi for 48 hours and stained to show CREST, CHMP7 and DAPI. (D-H) HeLa cells were treated with a $10 \mathrm{~mJ} / \mathrm{cm}^{2}$

764 dose of UV-C irradiation and fixed at various timepoints following treatment. The cells were stained for $\mathrm{YH} 2 \mathrm{AX}, \mathrm{CHMP7}$ and DAPI. (D) Accumulations of CHMP7 and $\mathrm{yH} 2 \mathrm{AX}$ within a single micronucleus in control cells and in cells 24 and 48 hours post-recovery from UV-C (scale bar $10 \mu \mathrm{m}$ ). The number of micronuclei per cell post accumulation and $(G) \mathrm{yH} 2 \mathrm{AX}$ foci, with micronuclei containing at least one focus

770 being considered as positive (400 cells per treatment). The percentage of 771 micronuclei positive for both $\mathrm{YH} 2 \mathrm{AX}$ and $\mathrm{CHMP7}$ are shown in $(\mathrm{H})$. The percentage 772 is calculated against the total number of micronuclei counted for each time point, 773 varying between a minimum of 50 and a maximum of 285. Averages and SEM are

774 shown $(\mathrm{N}=3)$. Results were analyzed using a one-way ANOVA with Dunnett's post 775 hoc test. 
777 Example of HeLa micronuclei positive or negative for RPA70 and CHMP4B. Scale

778 bar $3 \mu \mathrm{m}$. (B) Distribution of the status of CHMP7 and for presence or absence of 779 single-stranded DNA (RPA70) within the same micronucleus (minimum 115

780 micronuclei scored per repeat). (C) Examples of of HeLa micronuclei positive or negative for cGAS and CHMP7. Scale bar 3um. (D) Micronuclei in HeLa cells were scored for the status of CHMP7 and the status of cGAS within the same micronucleus (minimum 150 micronuclei scored per repeat). Data were analyzed using Fishers' exact test on pooled raw counts distributions $(\mathrm{N}=3)$. Scale bar $3 \mu \mathrm{m}$. (E) Micronuclei in HeLa cells transfected for $48 \mathrm{~h}$ with the indicated siRNA and scored for enrichment in RPA70 signal (minimum 50 total micronuclei scored per treatment, per repeat). Results were analyzed using a one-way ANOVA with

788 Dunnett's post hoc test. Averages and SEM shown (N=3). (F) Top panel: HeLa cells 789 depleted of CHMP7 for 48 hours (arrowheads show RPA70+ve micronuclei; filled 790 arrowheads show RPA70-ve micronuclei). Bottom panel: HeLa cells depleted of 791 VPS4 for 48 hours (arrowheads show RPA70+ve and CHMP4B+ve micronuclei).

792 Scale bar 10 $\mathrm{mm}$. (G) Micronuclei in HeLa cells transfected for $48 \mathrm{~h}$ with the indicated

793 siRNA and scored for enrichment in cGAS signal (minimum 55 micronuclei scored

794 per treatment, per repeat). Results were analyzed using a one-way ANOVA with

795 Dunnett's post hoc test. Averages and SEM shown $(\mathrm{N}=3) .(\mathrm{H})$ Top panel: HeLa cells

796 depleted of CHMP7 for 48 hours (arrowheads show cGAS+ve micronuclei; filled 
bioRxiv preprint doi: https://doi.org/10.1101/476630; this version posted December 1, 2018. The copyright holder for this preprint (which was not certified by peer review) is the author/funder, who has granted bioRxiv a license to display the preprint in perpetuity. It is made available under aCC-BY-NC-ND 4.0 International license.

797 arrowheads show cGAS-ve micronuclei). Bottom panel: HeLa cells depleted of VPS4

798 for 48 hours (arrowhead shows a cGAS+ve micronucleus; arrow shows cGAS

799 enrichment at a CHMP7 nuclear accumulation). Scale bar $10 \mu \mathrm{m}$. 


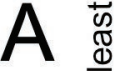

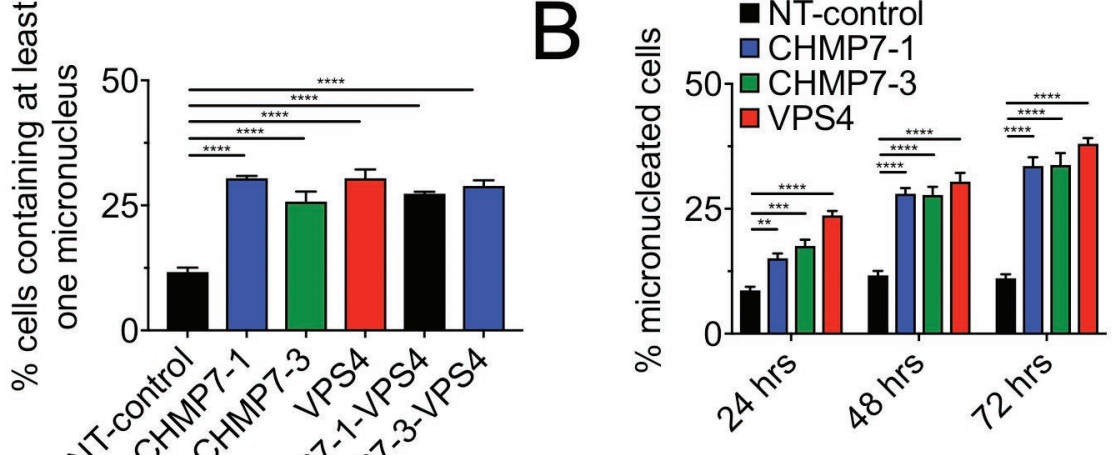

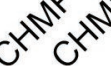
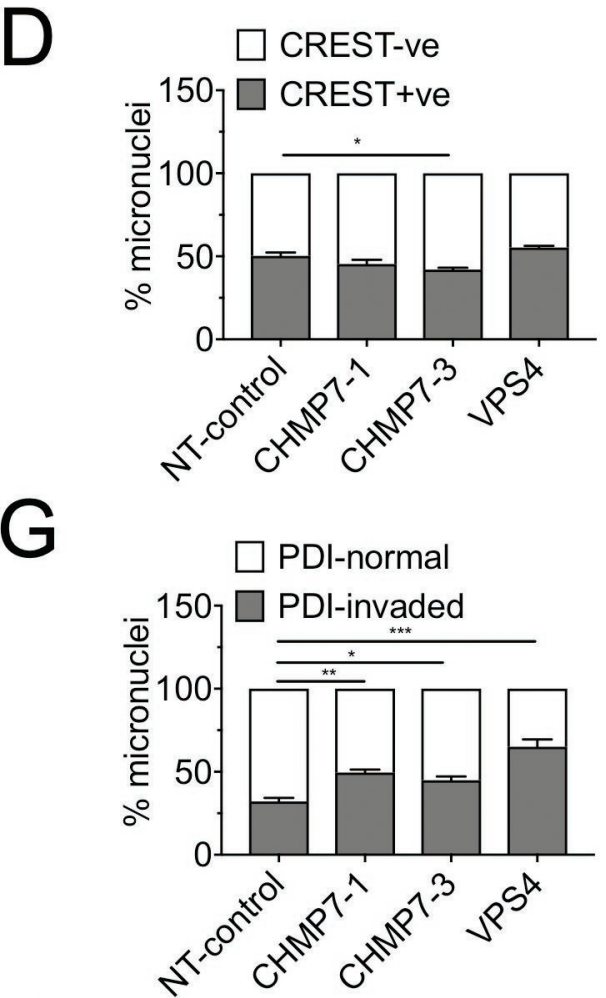

$\mid$
+
0
0
0
0

를
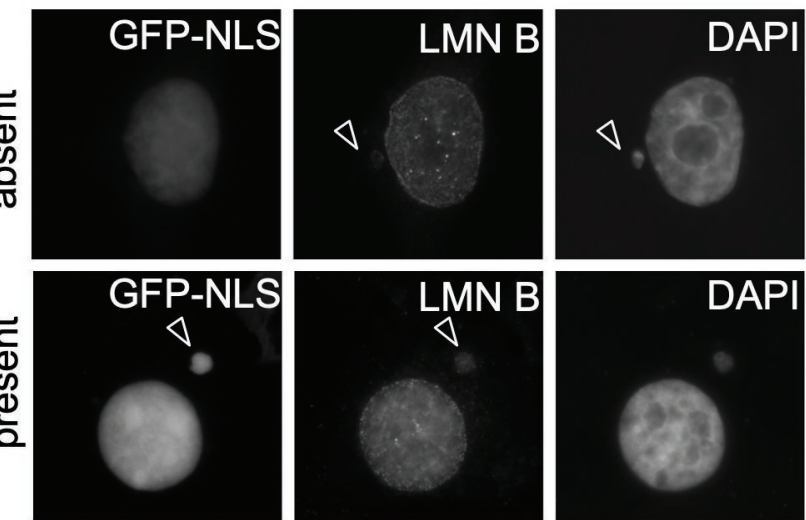

$\square$ LMNB1 continuous
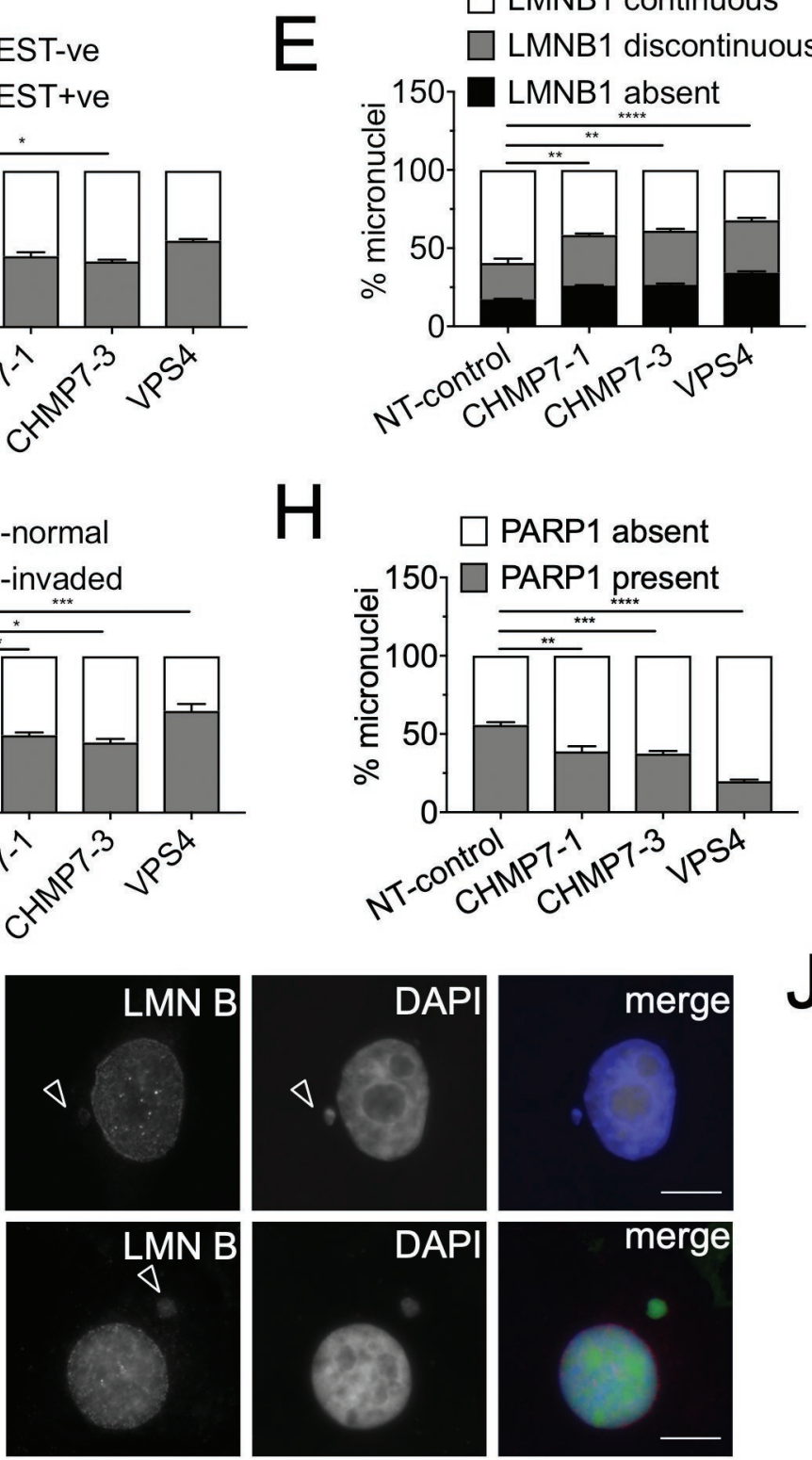

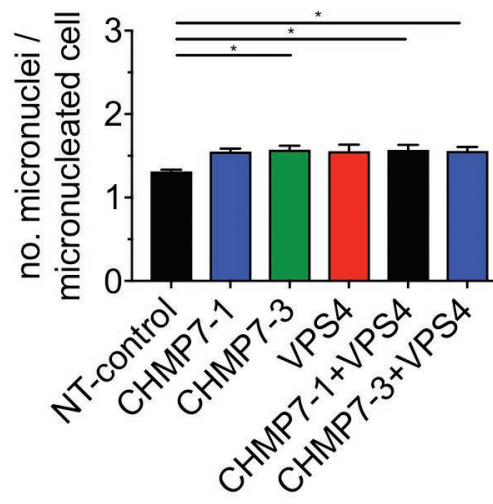

$\square$ mab414 normal

$\square$ mab414 reduced

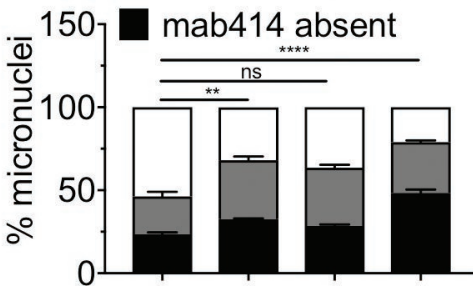

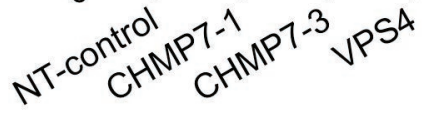

Figure 2. Impairment of ESCRT-III function affects micronuclei integrity.

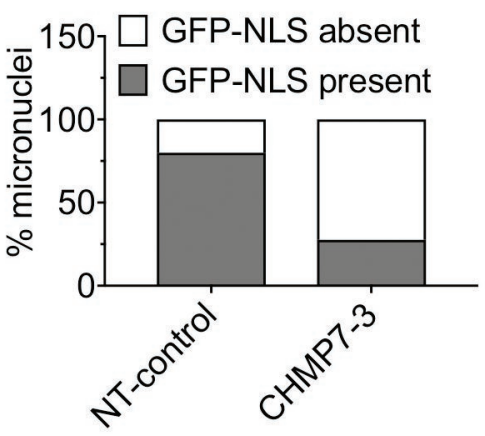



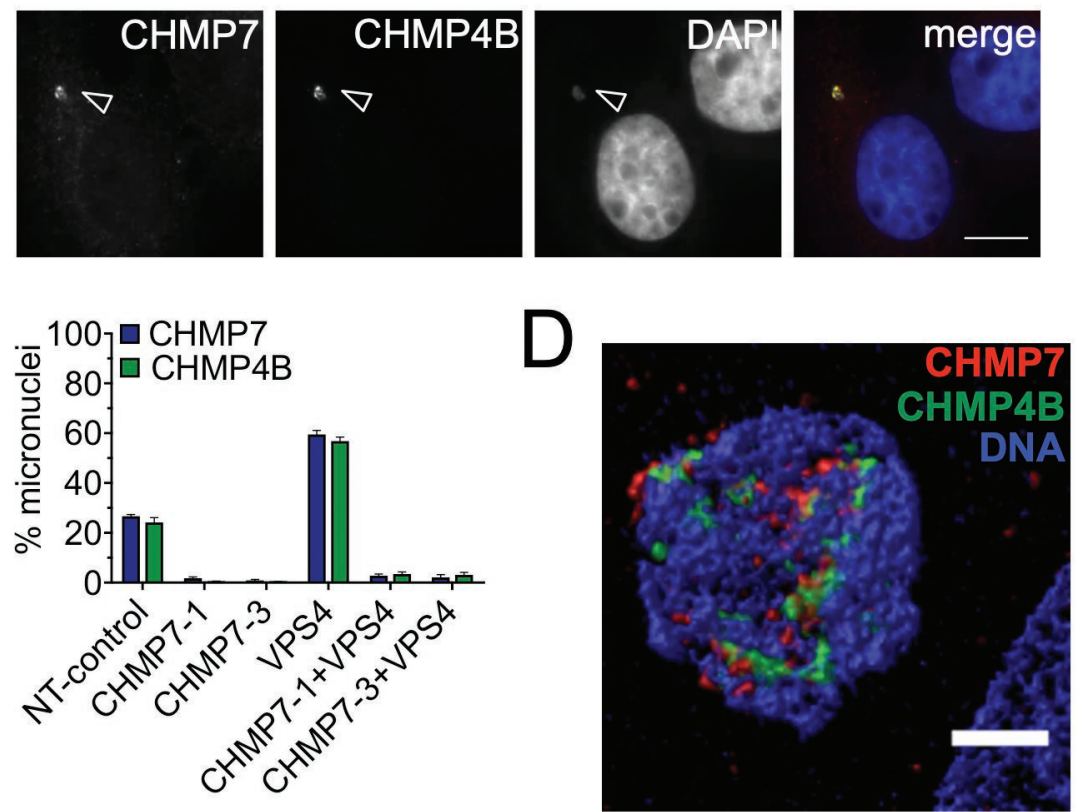

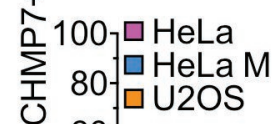
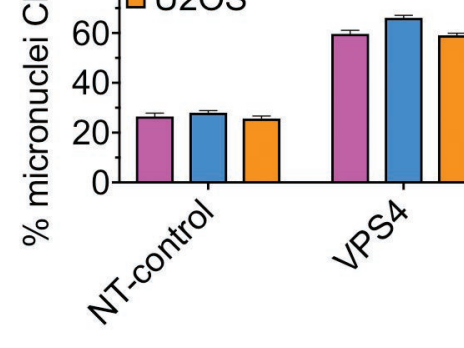

$E$
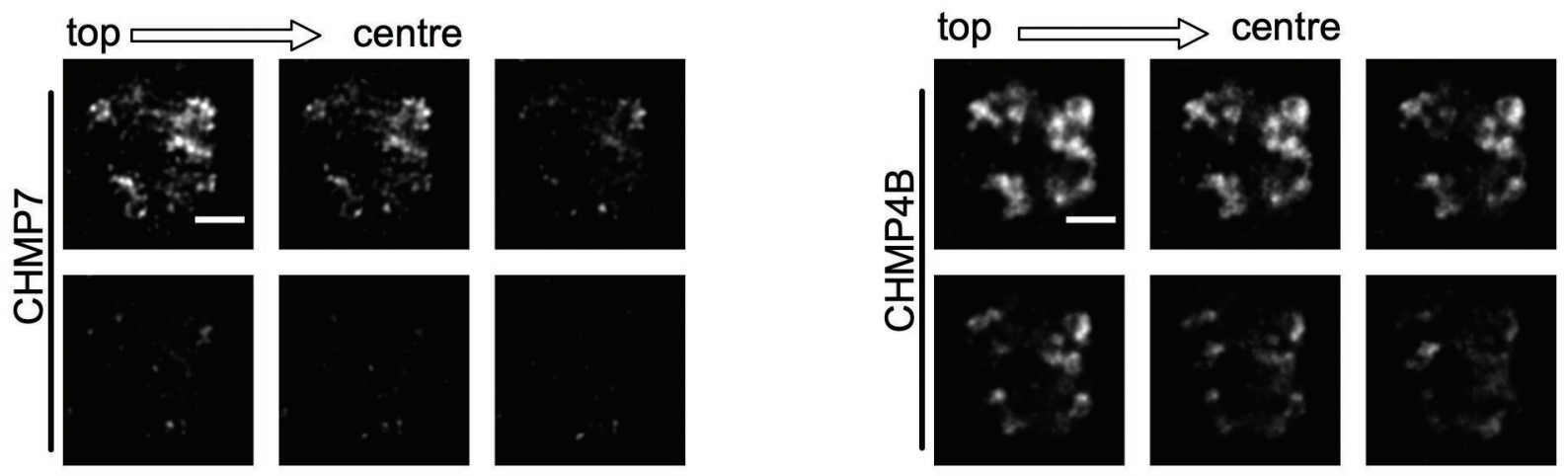

Figure 3. ESCRT-III accumulates on micronuclei and is recruited by CHMP7. 
A

CHMP7

PDI

DAPI

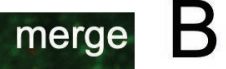

$p<.0001$

Cramer's V $=0.764$
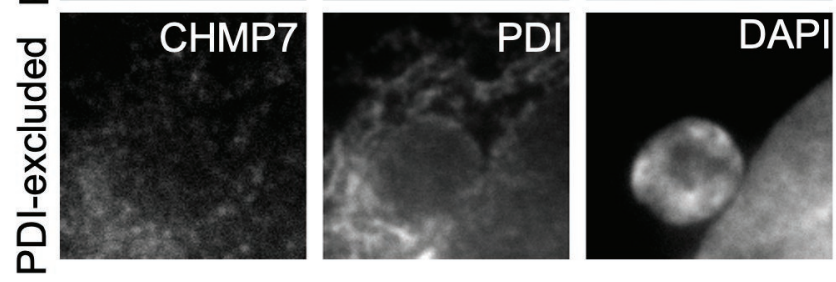

merge

150, $\square$ PDI-excluded

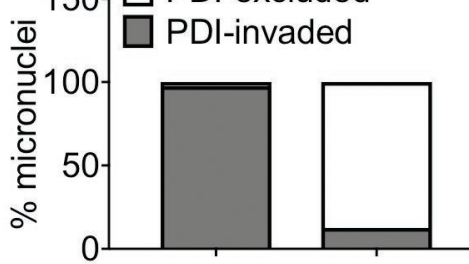

$c h M^{1+v e} c h p^{1^{-v e}}$
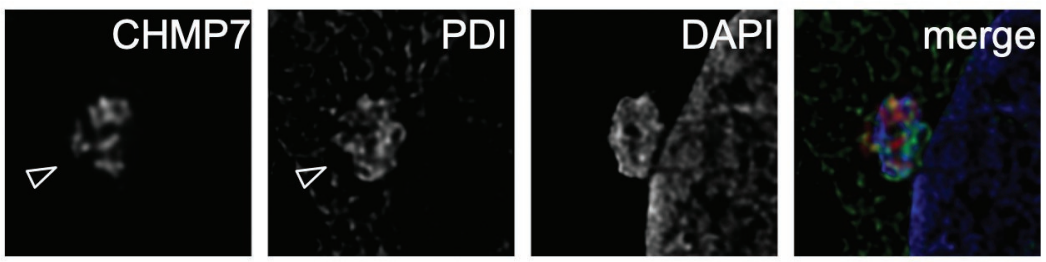

Figure 5. Micronuclei with ESCRT-III accumulations display ER membrane invasion. 

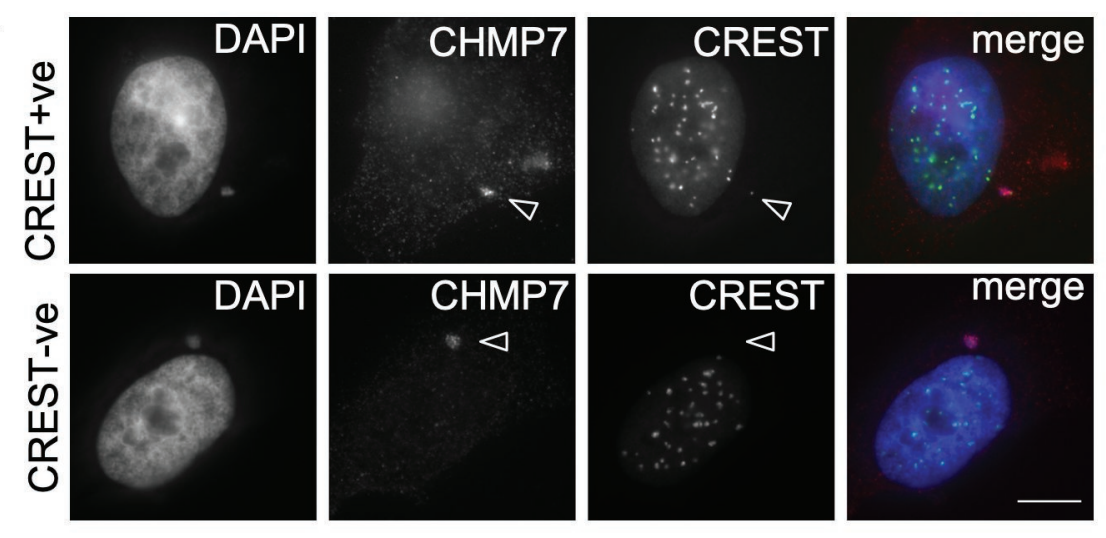

$p<.0001$

Cramer's V $=0.254$
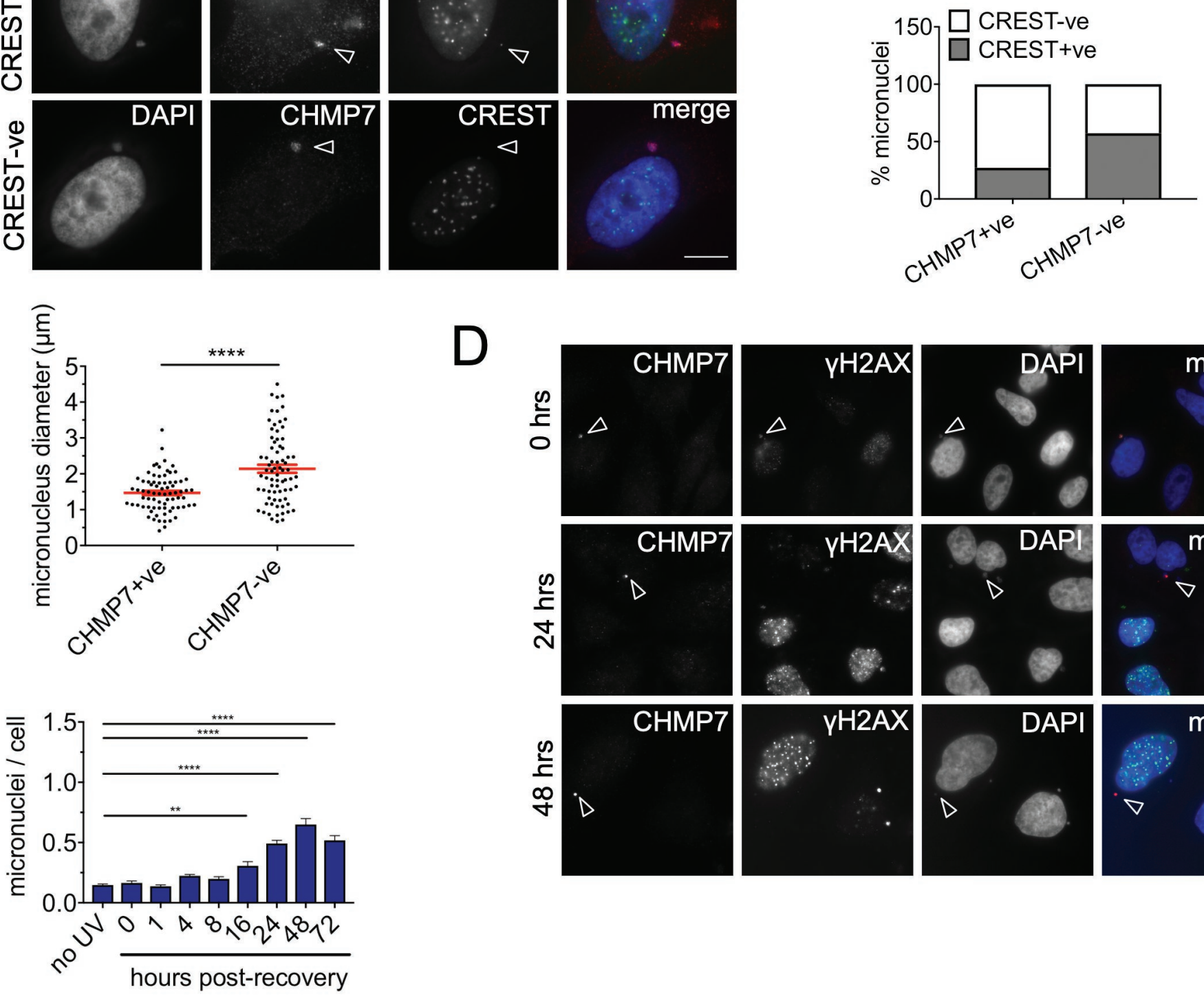

D
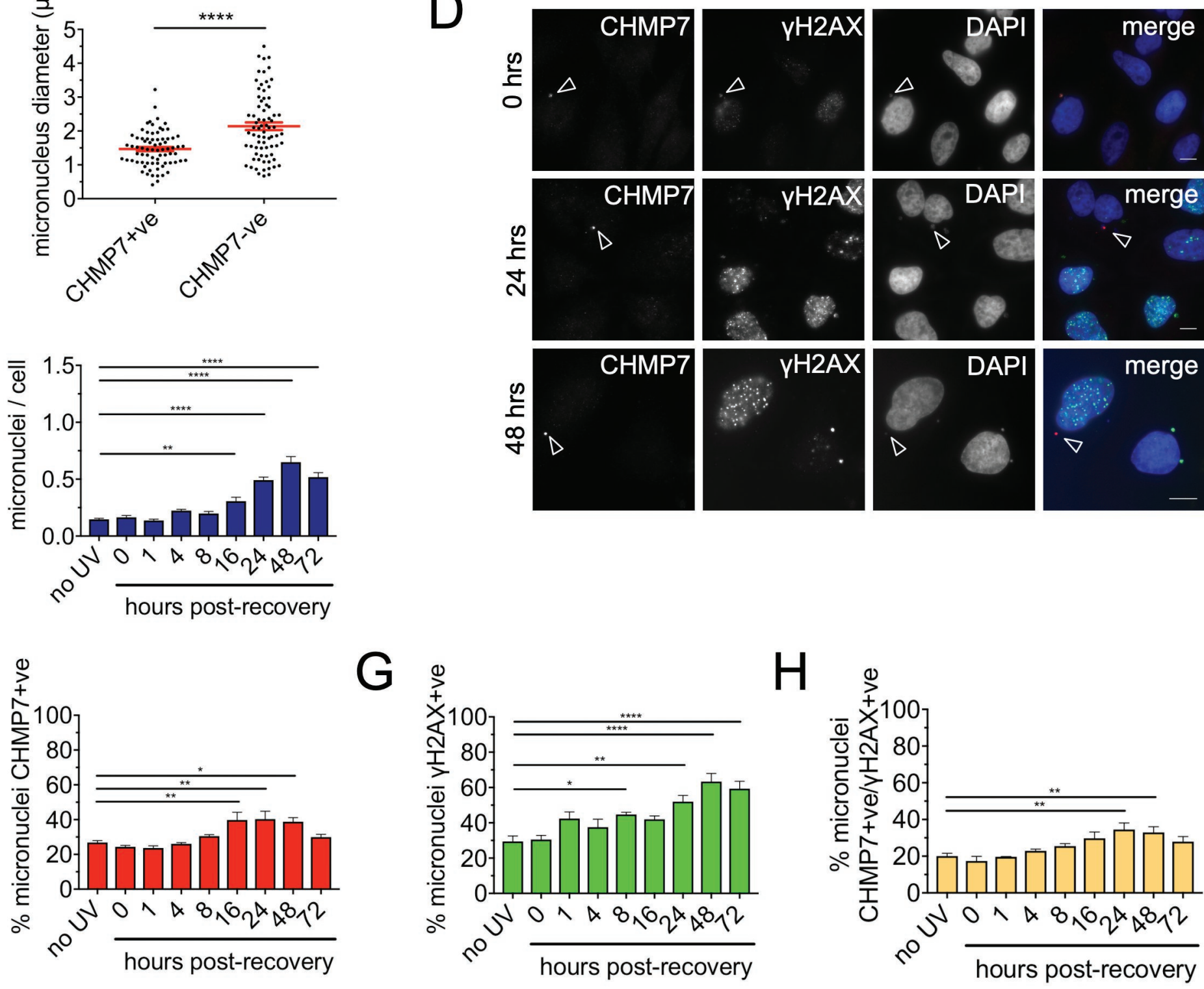

Figure 6. ESCRT-III preferentially accumulates on acentric micronuclei. 
A
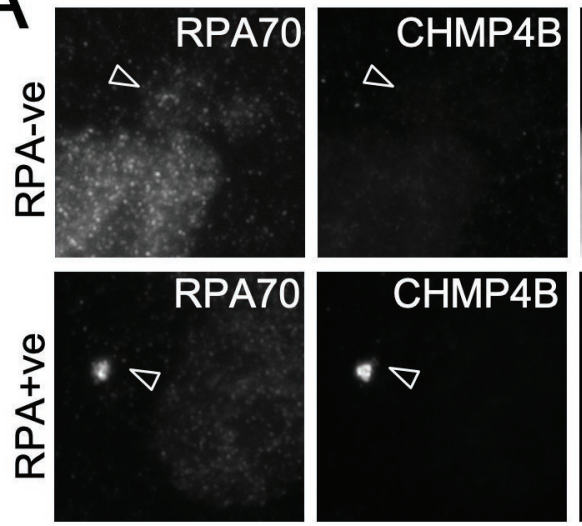

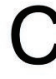

ن
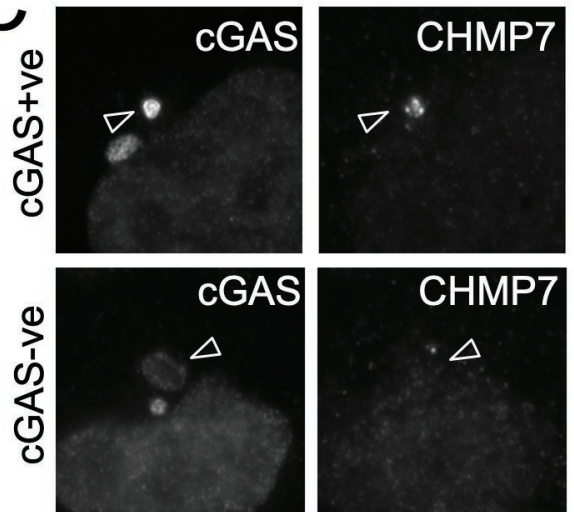

$\Delta$
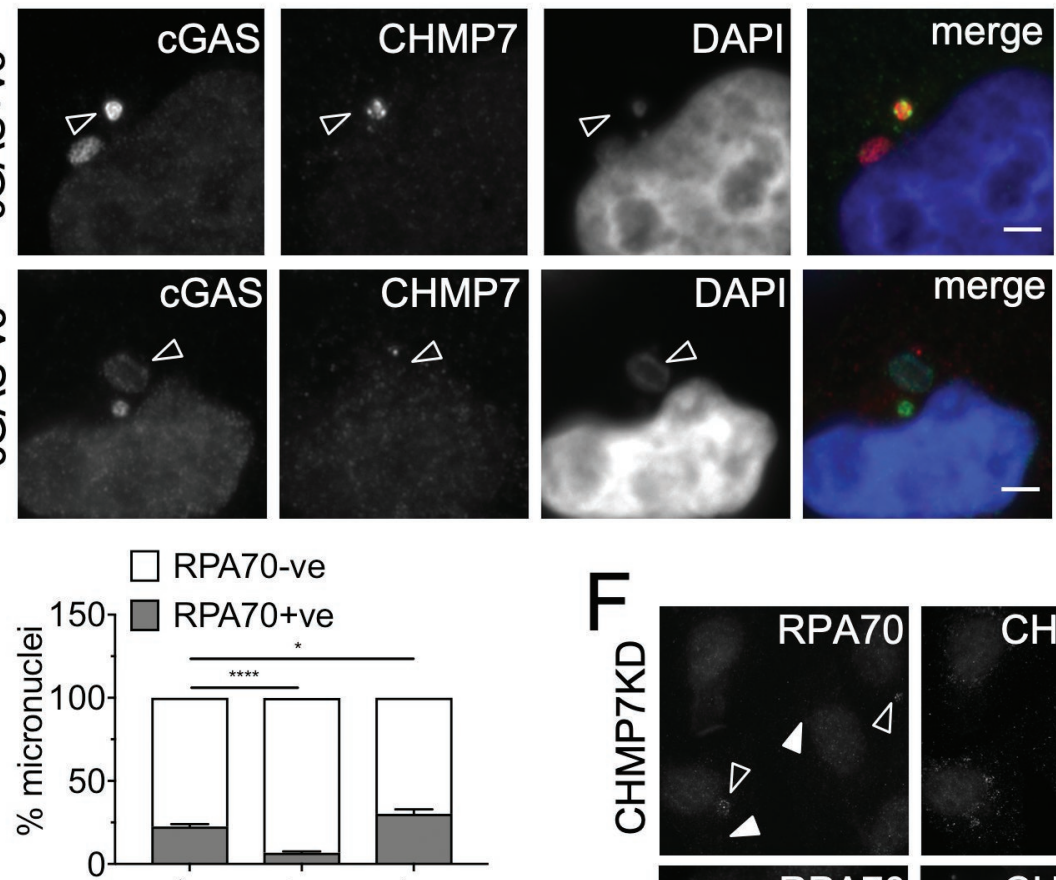

Control chMp N $^{-3}$ st $^{-3}$

5

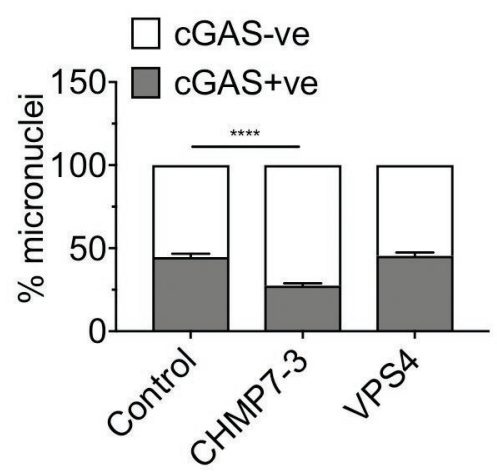

1
$D$

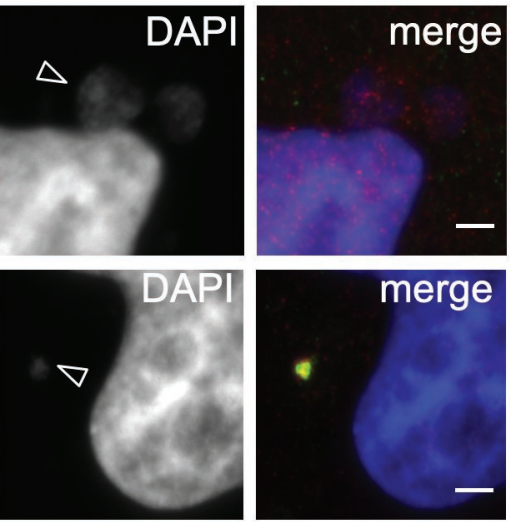

$p<.0001$

Cramer's V $=0.534$
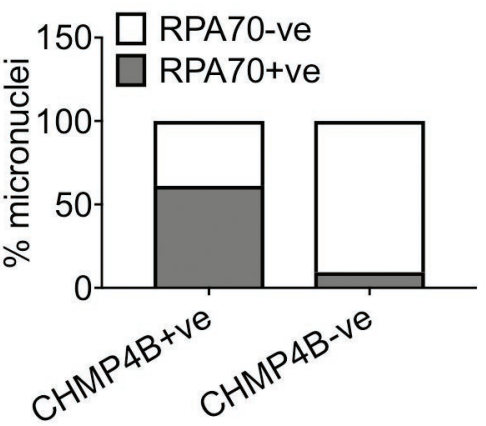

$p<.0001$

Cramer's V $=0.506$
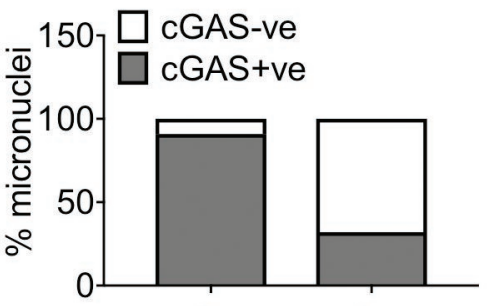

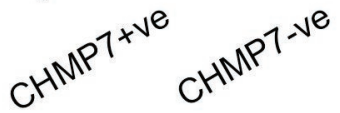
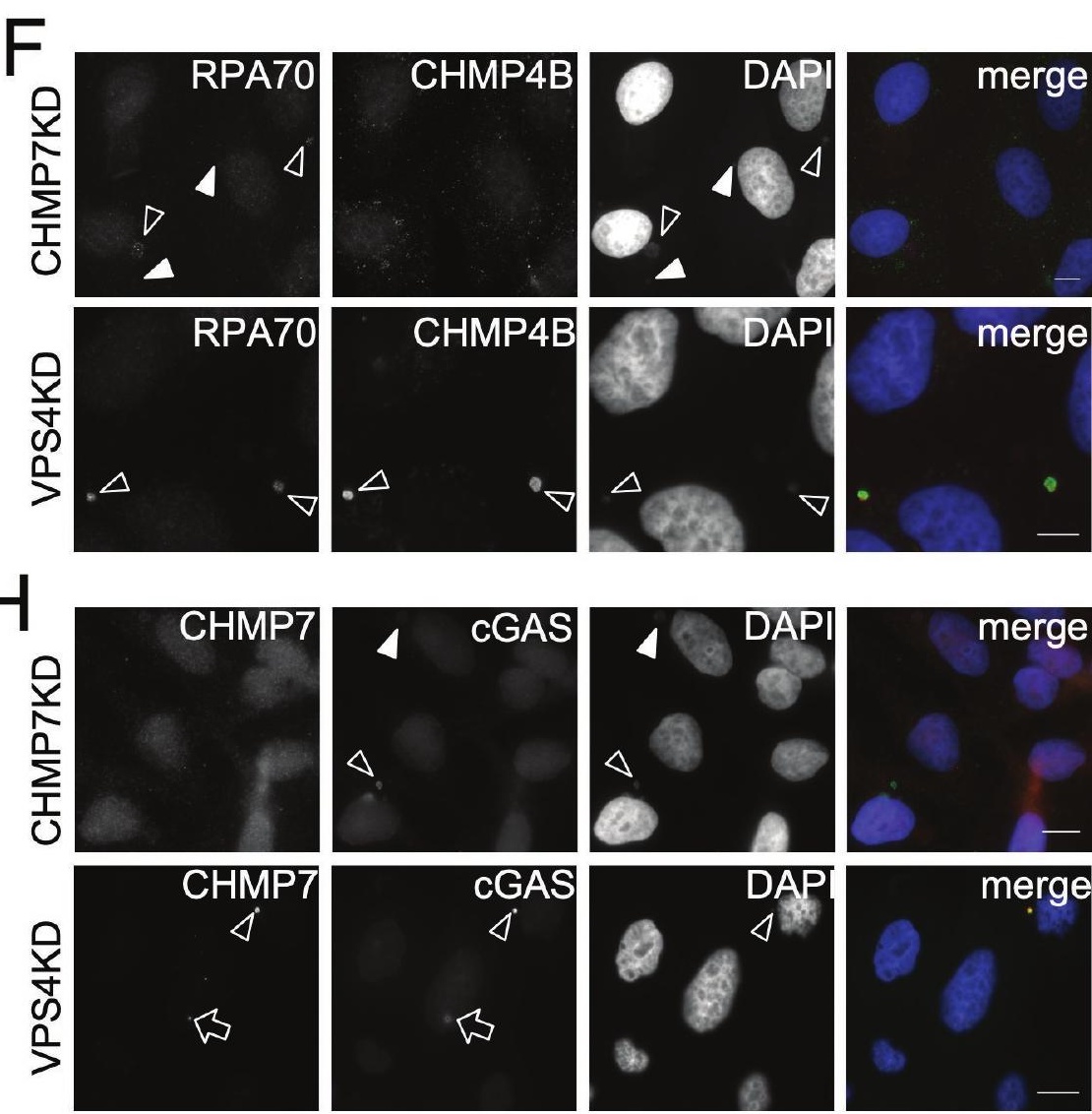

Figure 7. CHMP7 is important for generating damaged DNA at micronuclei. 